\title{
Cataclysmic Variables in Globular Clusters, the Galactic Center, and Local Space
}

\author{
Craig O. Heinke \\ University of Virginia, Astronomy Dept., PO Box 400325, Charlottesville VA 22903; \\ cheinke@virginia.edu \\ Ashley J. Ruiter \\ Dept. of Astronomy, New Mexico State University, 1320 Frenger Mall, Las Cruces, NM \\ 88003 \\ Michael P. Muno \\ Space Radiation Laboratory, California Institute of Technology, Pasadena, CA 91125 \\ Krzysztof Belczynski \\ Dept. of Astronomy, New Mexico State University, 1320 Frenger Mall, Las Cruces, NM \\ 88003; Tombaugh Fellow
}

\begin{abstract}
We compare the X-ray spectra and luminosities, in the 2-8 keV band, of known and suspected cataclysmic variables (CVs) in different environments, assessing the nature of these source populations. These objects include nearby CVs observed with ASCA; the Galactic Center X-ray source population identified by Muno et al.; and likely CVs identified in globular clusters. Both of the latter have been suggested to be dominated by magnetic CVs. We find that the brighter objects in both categories are likely to be magnetic CVs, but that the fainter objects are likely to include a substantial contribution from normal CVs. The strangely hard spectra observed from the Galactic Center sources reflect the high and variable extinction, which is significantly greater than the canonical $6 \times 10^{22} \mathrm{~cm}^{-2}$ over much of the region, and the magnetic nature of many of the brightest CVs. The total numbers of faint Galactic Center sources are compatible with expectations of the numbers of CVs in this field.
\end{abstract}

\section{Introduction}

The unprecedented spatial resolution of the Chandra X-ray Observatory allows us to study populations of faint X-ray sources at distances of kiloparsecs. Large numbers of X-ray sources of moderate luminosities $\left(10^{31}<L_{X}<10^{34} \mathrm{ergs} / \mathrm{s}\right)$ have been discovered in several Galactic environments (e.g. star-forming regions, globular clusters, the Galactic Center) in recent years. For instance, Grindlay et al. (2001); Pooley et al. (2002) and others have found large numbers of X-ray sources in globular clusters, many of which are optically identified as CVs. Muno et al. (2003) identified a large population of $\sim 2000$ faint, spectrally hard X-ray sources associated with the central $40 \mathrm{pc}$ around Sgr A*.

Interpretation of these observations in terms of known categories of X-ray sources is sometimes hampered by the different observing bands and models used for studies of nearby, well-known sources versus these distant populations. The Galactic Center sources have 
been inferred (Muno et al. 2003, 2004), by their X-ray luminosity and spectral hardness, to be composed largely of the class of CVs known as intermediate polars (Patterson 1994). Intermediate polars have white dwarf accretors with relatively high magnetic fields, which force the accreting material to follow the magnetic field lines of the accretor down onto the magnetic poles. The brightest CVs in globular clusters have been argued to be too X-ray luminous for normal CVs (Verbunt et al. 1997), and several pieces of evidence point to a magnetic nature of at least some CVs in globular clusters, including He II $\lambda 4686$ emission observed in NGC 6397 CVs (Grindlay et al. 1995), X-ray periodicities in some of the brightest CVs in 47 Tuc (Grindlay et al. 2001), enhanced $N_{H}$ columns towards the brightest CVs (Heinke et al. 2005), and the lack of dwarf nova outbursts from globular clusters (Shara et al. 1996; Edmonds et al. 2003; Dobrotka et al. 2006).

However, the question of whether magnetic CVs are more common than normal CVs in globular clusters or the Galactic Center has not yet been explored. We have directly compared, for the first time, the spectra of Galactic Center X-ray sources, and likely globular cluster CVs (those with optical counterparts), with archival ASCA X-ray spectra of wellknown nearby CVs. Full details will be published in Heinke et al. (2008, in prep).

\section{Local CVs}

We cross-correlated the CV database of Ritter \& Kolb (2003) ${ }^{1}$ with the ASCA observations in the HEASARC archive. We chose 20 confirmed IPs with substantial pointed ASCA observations, 11 polars, 8 novalike (or outbursting dwarf nova) CVs, and 11 quiescent dwarf nova observations (see Baskill et al. 2005; Ezuka \& Ishida 1999). We used the archived spectra and responses from the ASCA archive, and extracted appropriate background spectra.

We fit the ASCA spectra from 2-8 keV, in order to compare the same energy range for all our data. A power-law model with a single gaussian (representing the combination of Fe lines) generally gave acceptable fits to the data. We find (omitting $3 \mathrm{CVs}$ with poorly determined indices) a clear difference between the fitted photon indices of the magnetic (mean $\Gamma=1.22, \sigma=0.33$ ) and the nonmagnetic (mean $\Gamma=1.97, \sigma=0.20$ ) ASCA CVs. We find consistency, however, between the average IP and polar spectra, and between the average quiescent dwarf nova spectrum and the average novalike/outbursting dwarf nova spectrum. High- $B$ IPs and polars (e.g. AE Aqr and V884 Her) have suppressed hard X-ray radiation, producing low $L_{X} \mathrm{~s}$ and photon indices. Excluding these high- $B$ systems, we do not find strong dependence of the photon index with $L_{X}$ within either the magnetic CVs or nonmagnetic CVs as groups.

\section{Globular Cluster CVs}

For comparison with the ASCA observations and Galactic Center sources, we perform homogeneous X-ray spectral fitting of 23 (optically) identified CVs in globular clusters (47 Tuc, NGC 6397, $\omega$ Cen, NGC 6752, and M4), with >90 counts above $2 \mathrm{keV}$. We compare the fitted power-law photon indices and estimated X-ray luminosities of the globular cluster CVs with the observed CV populations in Figure 1a. The locations of globular cluster X-ray sources in this plot suggest that some (the brightest and hardest) are likely magnetic systems, while many others are likely nonmagnetic systems.

${ }^{1}$ http://physics.open.ac.uk/RKcat/ 

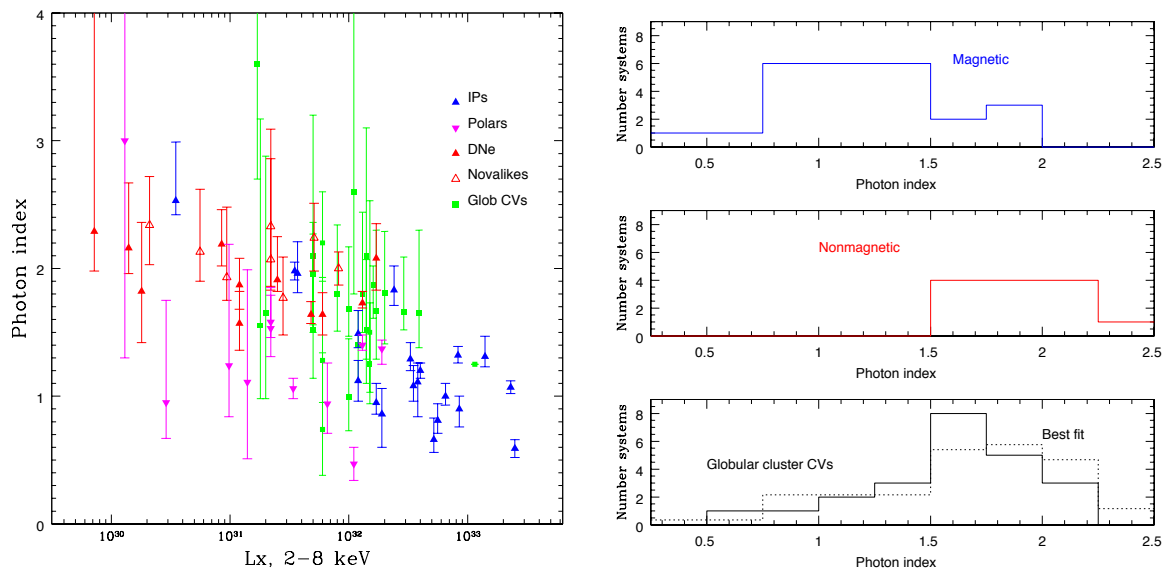

Figure 1. Left: X-ray luminosities vs. photon index measurements for various classes of nearby CVs vs. those measured for globular cluster X-ray sources optically identified as CVs. Right: Histograms of the photon indices of magnetic, nonmagnetic, and globular cluster CVs. The bottom panel also shows the best-fit distribution of photon index produced by scaling the histograms of magnetic and nonmagnetic $\mathrm{CV}$ photon indices to match the globular cluster CVs.

We selected an X-ray luminosity range $\left(10^{31}\right.$ to $\left.2 \times 10^{33} \mathrm{ergs} / \mathrm{s}\right)$ that includes all globular cluster CVs in our list. In this range we find 25 magnetic systems and 12 nonmagnetic systems with ASCA spectra. We show histograms of the photon indices of magnetic, nonmagnetic, and cluster CVs in Fig. 1b. We scaled the histograms of magnetic and nonmagnetic CV indices to match the histogram of globular cluster CV indices. We find a best fit of $39_{-15}^{+12}(1 \sigma) \%$ magnetic systems, i.e. 5 to 12 of the 23 confirmed globular cluster CVs, with the rest being nonmagnetic systems. Since these systems have been identified in a nonuniform way, with strong X-ray selection, it is likely that the fraction of magnetic CVs in globular clusters is lower than this value. We do not find evidence that the fraction of magnetic CVs (polars and intermediate polars) in globular clusters is higher than the $\sim 10 \%$ estimated for the field (Liebert et al. 2003).

\section{Galactic Center Sources}

Muno et al. (2004) characterize the Galactic Center sources, finding typical X-ray luminosities of $3 \times 10^{31}-10^{33} \mathrm{ergs} / \mathrm{s}$, and very hard X-ray spectra, with equivalent photon indices generally between 1 and -1 . This is rather harder than the typical globular cluster CV or field CV, or even the magnetic CVs in either location (Heinke et al. 2006). Muno et al. (2004) pointed out that selection effects (the high extinction and diffuse background) probably had a role in the hardness of these sources. To test whether the Galactic Center sources were consistent with a combination of magnetic and nonmagnetic CVs, or even with just magnetic CVs, we undertook MARX simulations in which we added sources of known properties to $414 \mathrm{ks}$ (2/3 of the total used by Muno et al. (2003); the 3 longest observations, to re- 

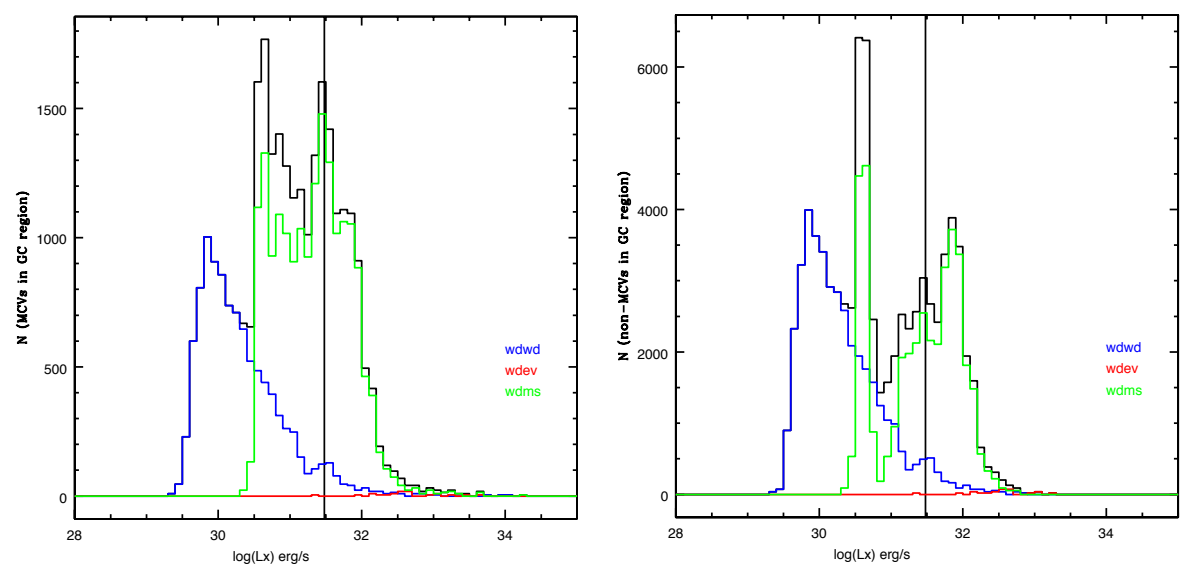

Figure 2. Left: Histograms of $L_{X}(2-8 \mathrm{keV})$ from StarTrack population synthesis for magnetic CVs. Blue: white dwarf-white dwarf systems, green: white dwarf-main sequence systems, red: white dwarf-evolved star systems. Black vertical line: rough lower limit of Muno observations. Right: Same as left, but for nonmagnetic CVs.

duce computing time) of the real Chandra observations of the Galactic Center, ran detection algorithms and measured the colors of the detected fake sources.

For our simulated source population, we choose a population synthesis model using the StarTrack code (Belczynski et al. 2008) as implemented in Ruiter et al. (2006), with updates to compute the X-ray luminosities of magnetic and nonmagnetic CVs using the prescription of Patterson \& Raymond (1985). We show the luminosity functions for magnetic and nonmagnetic systems in Figure 2. We assume that $10 \%$ of the total CV population are magnetic systems.

For the spectra of the simulated CVs, we choose absorbed power-law spectra with single gaussians to represent the $\mathrm{Fe} \mathrm{K}$ line complex, with average energies and equivalent widths based on the ASCA fits. Our absorption includes both photoelectric absorption (using the XSPEC model phabs) and scattering by dust (using P. Predehl's XSPEC model scatter, Predehl et al. (2003)). For nonmagnetic systems, we use an average photon index of 1.97. For magnetic systems, we produce simulated systems with photon indices of $0.72,1.22$, and 1.72 , with a distribution set by the results from the ASCA magnetic CV spectra. The only parameter we adjust to match the observations is the extinction. We compare the simulated systems to the real sources, extracted in the same way from the same Galactic Center data. We compare the medium and hard colors (defined by Muno et al. 2003, as (h-s)/(h+s), where the medium color uses the 2.0-3.3 and 3.3-4.7 keV bands, and 3.3-4.7 and 4.7-8 for the hard color) and measured photon fluxes (see Figure 3).

We find that the real data can be reasonably described with our model only if a higher $N_{H}$ of $10^{23} \mathrm{~cm}^{-2}$ (plus dust scattering) absorbs the majority of the sources, rather than the canonical $N_{H}=6 \times 10^{22}$. Figures 3 (color-flux) and 4 (color-color) show samples of our results, using only $N_{H}=6 \times 10^{22}$ (left panels) or $10^{23} \mathrm{~cm}^{-2}$ (right panels). It can be seen 

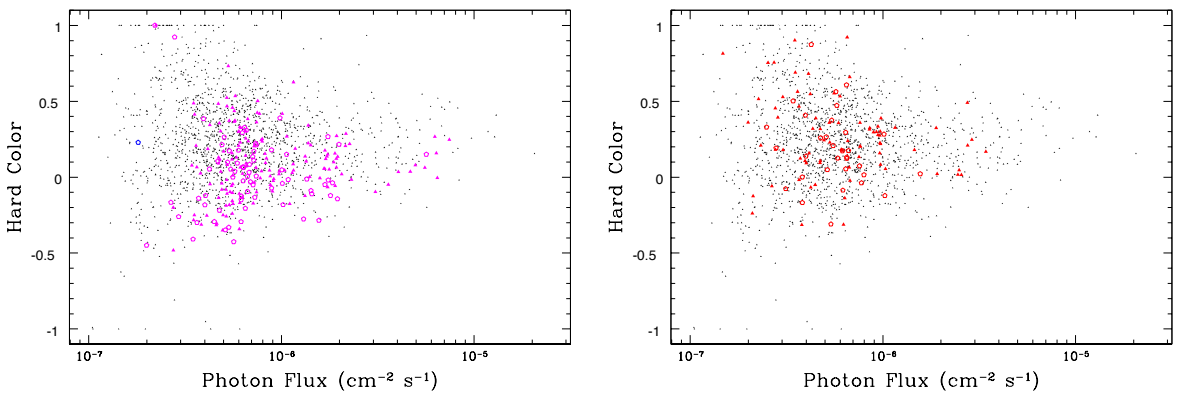

Figure 3. Photon flux vs. hard color for Galactic Center sources (black), and for our simulations with $N_{H}=6 \times 10^{22}$ (left, magenta) or $10^{23} \mathrm{~cm}^{-2}$ (right, red). Definitions of photon flux and hard color are the same as in Muno et al. (2004). Triangles indicate simulations of magnetic systems, open pentagons nonmagnetic systems.
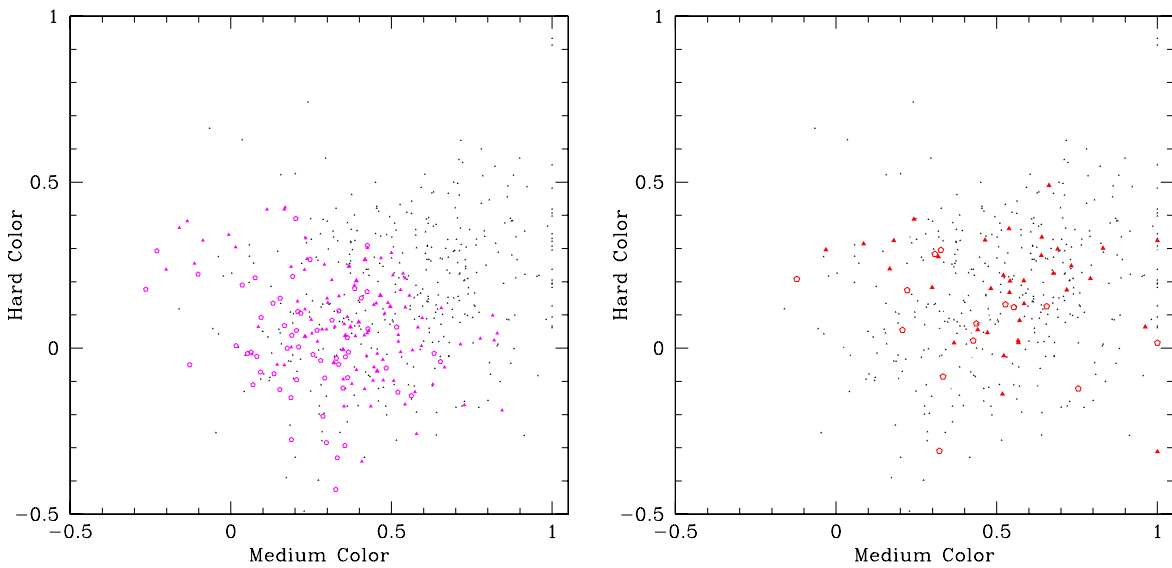

Figure 4. Medium color vs. hard color for Galactic Center sources (black) and for our simulations with $N_{H}=6 \times 10^{22}$ (left, magenta) or $10^{23} \mathrm{~cm}^{-2}$ (right, red). Definitions of colors as in Muno et al. (2003); triangles indicate simulations of magnetic systems and open pentagons nonmagnetic systems. 


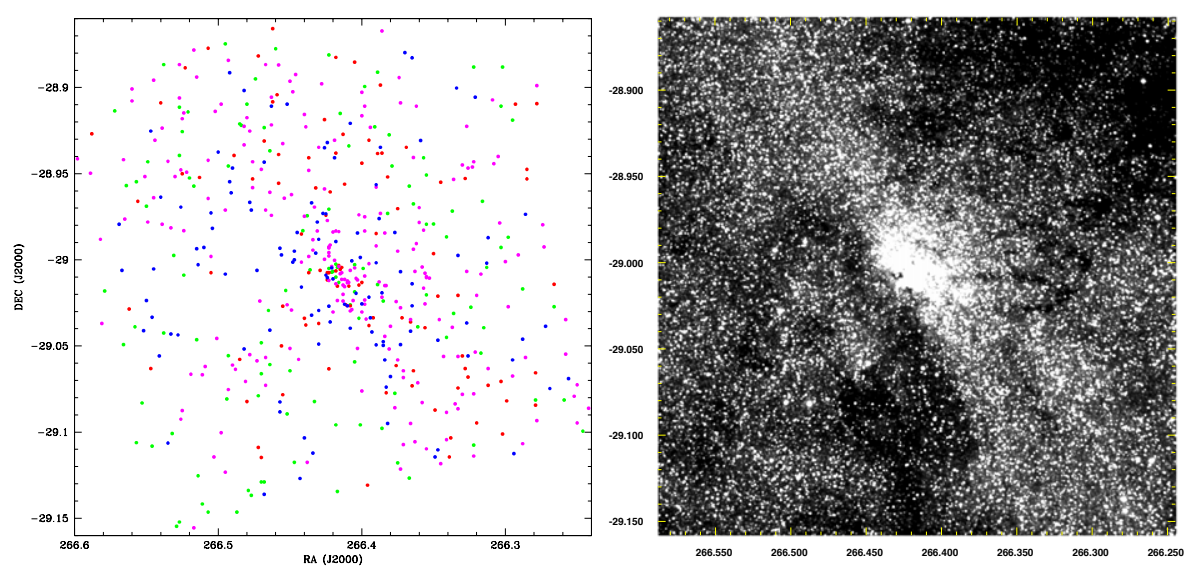

Figure 5. Left: Locations of Chandra X-ray sources from Muno et al. (2004), coded by the $N_{H}$ value in fits to thermal plasma spectra. Green: $N_{H}<6 \times 10^{22}$, red: $6-10 \times 10^{22}$, magenta: $10-20 \times 10^{22}$, blue: $>20 \times 10^{22}$. Right: K-band image of Galactic Center, from the 2MASS survey.

that the right panels exhibit much better qualitative matches to the data. Similar agreement can be reached using broader ranges of $N_{H}$ with an average extinction of $10^{23} \mathrm{~cm}^{-2}$.

The interstellar absorption towards the Galactic Center is known to be inhomogeneous and filamentary. The variations in this absorption have a strong effect on the numbers and hardness of the galactic center X-ray sources seen at different positions. This can be qualitatively seen in Figure 5, which compares a K-band image of the Galactic Center from the 2MASS survey with the positions and fitted $N_{H}$ values of Galactic Center X-ray sources. Current near-IR observations of the Galactic Center field (e.g. Gosling et al. 2006) will improve our understanding of the effects of extinction upon X-rays from the Galactic Center.

The total number of CVs in the Galactic Center may be inferred from our results (with caveats, particularly the variability of extinction in the region). The total number of simulated CVs required to match the numbers of observed sources, using a single extinction of $N_{H}=10^{23} \mathrm{~cm}^{-2}$, is about 7000. Following Muno et al. (2003), and using an estimate of $1 \times 10^{-5} \mathrm{CVs} \mathrm{pc}^{-3}$ (Grindlay et al. 2005) in local space, we estimate a total CV number in the Galactic Center of 5000. This numerical agreement indicates that CVs are indeed the major contributor to the Galactic Center X-ray sources, and suggests that these CVs are not significantly different in their X-ray properties or formation mechanisms from CVs in our galactic neighborhood.

\section{Conclusions}

Studies of faint $\left(10^{31}<L_{X}<10^{34} \mathrm{ergs} / \mathrm{s}\right)$ hard X-ray sources in globular clusters and the Galactic Center have identified them as primarily CVs, on both observational and theoretical 
(lack of a sufficiently numerous alternative population) grounds. They have also suggested that many or most of them are magnetic systems, particularly intermediate polars. We have compared the spectra of nearby CVs, observed with ASCA, to the low-count spectra or colors of globular cluster CVs and Galactic Center X-ray sources. We find that significant (although poorly constrained) fractions of the observed cluster and galactic center populations are consistent in their X-ray spectra and fluxes with nonmagnetic CVs. For the Galactic Center, we require a somewhat higher average extinction than typically assumed $\left(N_{H}=10^{23}\right.$ instead of $6 \times 10^{22} \mathrm{~cm}^{-2}$ ). We do not find evidence for significant differences between the X-ray properties and fraction of magnetic systems of CVs in local space, vs. those of CVs in globular clusters or the Galactic Center.

Acknowledgments. We thank R. E. Taam and Koji Mukai for useful conversations. $\mathrm{COH}$ has been supported by the Lindheimer Fellowship at Northwestern University, and Chandra grant G07-8078X at the Univ. of Virginia while doing this work. MPM has been supported by a Hubble Fellowship, while KB has been supported by a Tombaugh Fellowship. AJR was supported by a Chandra Theory Grant and is thankful to the Dept. of Physics and Astronomy at Northwestern University for their hospitality. This publication makes use of data products from the Two Micron All Sky Survey, which is a joint project of the University of Massachusetts and the Infrared Processing and Analysis Center, funded by the National Aeronautics and Space Administration and the National Science Foundation.

\section{References}

Baskill, D. S., Wheatley, P. J., \& Osborne, J. P. 2005, MNRAS, 357, 626

Belczynski, K., Kalogera, V., Rasio, F. A., et al. 2008, ApJS, 174, 223

Dobrotka, A., Lasota, J.-P., \& Menou, K. 2006, ApJ, 640, 288

Edmonds, P. D., Gilliland, R. L., Heinke, C. O., \& Grindlay, J. E. 2003, ApJ, 596, 1197

Ezuka, H., \& Ishida, M. 1999, ApJS, 120, 277

Gosling, A. J., Blundell, K. M., \& Bandyopadhyay, R. 2006, ApJ, 640, L171

Grindlay, J. E., Cool, A. M., Callanan, P. J., Bailyn, C. D., Cohn, H. N., \& Lugger, P. M. 1995, ApJ, 455, L47

Grindlay, J. E., Heinke, C., Edmonds, P. D., \& Murray, S. S. 2001, Science, 292, 2290

Grindlay, J. E., Hong, J., Zhao, P. et al. 2005, ApJ, 635, 920

Heinke, C. O., Grindlay, J. E., Edmonds, P. D., et al. 2005, ApJ, 625, 796

Heinke, C. O., Wijnands, R., Cohn, H. N., Lugger, P. M., Grindlay, J. E., Pooley, D., \& Lewin, W. H. G. 2006, ApJ, 651, 1098

Liebert, J., Bergeron, P., \& Holberg, J. B. 2003, AJ, 125, 348

Muno, M. P., Arabadjis, J. S., Baganoff, F. K., et al. 2004, astro-ph/0403463

Muno, M. P., Baganoff, F. K., Bautz, M. W., et al. 2003, ApJ, 589, 225

Patterson, J. 1994, PASP, 106, 209

Patterson, J., \& Raymond, J. C. 1985, ApJ, 292, 535

Pooley, D., Lewin, W. H. G., Homer, L., et al. 2002, ApJ, 569, 405

Predehl, P., Costantini, E., Hasinger, G., \& Tanaka, Y. 2003, AN, 324, 73

Ritter, H., \& Kolb, U. 2003, A\&A, 404, 301

Ruiter, A. J., Belczynski, K., \& Harrison, T. E. 2006, ApJ, 640, L167

Shara, M. M., Bergeron, L. E., Gilliland, R. L., Saha, A., \& Petro, L. 1996, ApJ, 471, 804

Verbunt, F., Bunk, W. H., Ritter, H., \& Pfeffermann, E. 1997, A\&A, 327, 602 